\title{
Brittle Fracture-Based Experimental Methodology for Microstructure Analysis
}

\author{
J.P.M. Hoefnagels ${ }^{1, a}$, C.C. Tasan ${ }^{1,2}$, M. Pradelle ${ }^{3}$, M.G.D. Geers ${ }^{1}$ \\ ${ }^{1}$ Eindhoven University of Technology, Faculty of Mechanical Engineering, \\ Section of Materials Technology, 5600MB, Eindhoven, the Netherlands \\ ${ }^{2}$ Materials Innovation Institute (M2i), PO Box 5008, 2600 GA Delft, the Netherlands \\ ${ }^{3}$ Polytech'Grenoble, Departement Materiaux P.O.Box 53, 38041 Grenoble, France \\ ${ }^{a}$,j.p.m.hoefnagels.tue.nl
}

Keywords: metallography, experimental methodology, specimen preparation, microscopic analysis, brittle fracture

\begin{abstract}
Metallographic techniques are crucial tools for establishing the connection between observed mechanical behaviour of metals and the underlying mechanisms in their microstructures. In this work, we propose a methodology that minimizes deformation and/or modification of the microstructure during specimen preparation, and provides a 3D representation of the deformed micromorphology. This methodology involves opening up fractured (tensile test) specimens under the ductile-to-brittle transition temperature of metals to yield two parts in a brittle manner. The occurrence of brittle fracture is validated by analyzing the detailed, unaltered microstructure from SEM characterization and surface profilometry mapping of sets of two parts. It is found that this technique yields additional valuable information regarding the size and morphology of deformed grains or nucleation mechanisms of ductile damage. Experiments with a number of different steels show that the methodology can be applied to sheet of different formability.
\end{abstract}

\section{Introduction}

The global response of a metal under mechanical loading is determined by the underlying physical events taking place locally as a response to that load. These physical events, or mechanisms, can only be studied if the microstructure of the metal can be analyzed at different stages of deformation. Unfortunately, the major fraction of the microstructure is 'inside', i.e. confined within the surface of, the metal. Several specimen preparation tools and protocols are developed during the last century to analyze this hidden portion of the microstructure. The general strategy in all of these methodologies involves the removal of the layer 'in front of' the cross section of interest, either mechanically (e.g. grinding, polishing), chemically (e.g. etching), electrochemically (e.g. electropolishing, electro- discharge machining) or physically (e.g. focused ion beam (FIB)). Although material removal can be achieved with acceptable precision with each of these processes, reports in the literature show that the microstructure in the cross section of interest is also modified, and in some cases deformed significantly. While mechanical processes cause subsurface hardening [1,2] and smearing effects [3], chemical and electrochemical processes by definition round off sharp edges (e.g. edges of the specimen, microvoids etc.) effectively altering the dimensions of the microstructural features. Even for FIB processing, with which material removal can be carried out very accurately, recent studies shows that significant amounts of $\mathrm{Ga}$ ions are embedded in the matrix of the metal being processed [4]. As these effects are well known in the world of metallography, lengthy specimen preparation protocols are generally designed to limit these effects to acceptable levels. Such procedures, however, are very unpractical, expensive and yet still nonconclusive, as it is experimentally very difficult to determine the level of deformation, alteration or modification due to a preparation process. Moreover, regardless of the type of the tool itself, the 
material that is removed is "lost", such that no data can be obtained from the other side of the examined microstructure.

Recent technological developments brought another group of experimental tools to the service of material scientists. Non-destructive tomography techniques (e.g. x-ray, atom, and electron) allow the analysis of the 3D microstructure of materials. However, there are still many limitations to overcome in this field, both concerning technical (e.g. resolution, specimen size) and practical (e.g. availability) aspects. Furthermore, significant density differences are necessary to resolve different features of a microstructure, which limits the use for characterization of e.g. microstructural details of polycrystalline metals.

In this work we scrutinize a different methodology, which involves no mechanical, chemical or physical process to reveal the cross section of interest $[5,6]$. Instead, a mechanical characteristic of the metal itself, ductile-to-brittle transition, is used such that the specimens are brittle-fractured to two pieces along the cross section of interest to open up the specimen for investigation. However, brittle fracture is commonly referred to as "catastrophic failure", mainly due to its sudden and difficult to control nature. To control brittle fracture in the desired path an experimental setup is developed, which enforces the brittle fracture of a specimen - e.g. a tensile specimen that was tested up to fracture - at any cross section of choice. The applicability of this methodology is tested using three different sheet metals. Obtained results show that this methodology is very promising for fast, practical, but also accurate characterization of metal microstructures

\section{Experimental Methodology}

The most important factors that play a role in ductile-to-brittle transition are stress intensification, strain rate and temperature [7]. It is known that especially at high stress triaxiality, high strain rate and low temperatures brittle fracture is generally more favorable to ductile fracture. A special methodology has been designed accordingly, that maximizes the possibility of brittle fracture in a controlled manner, which is shown in Fig. 1.
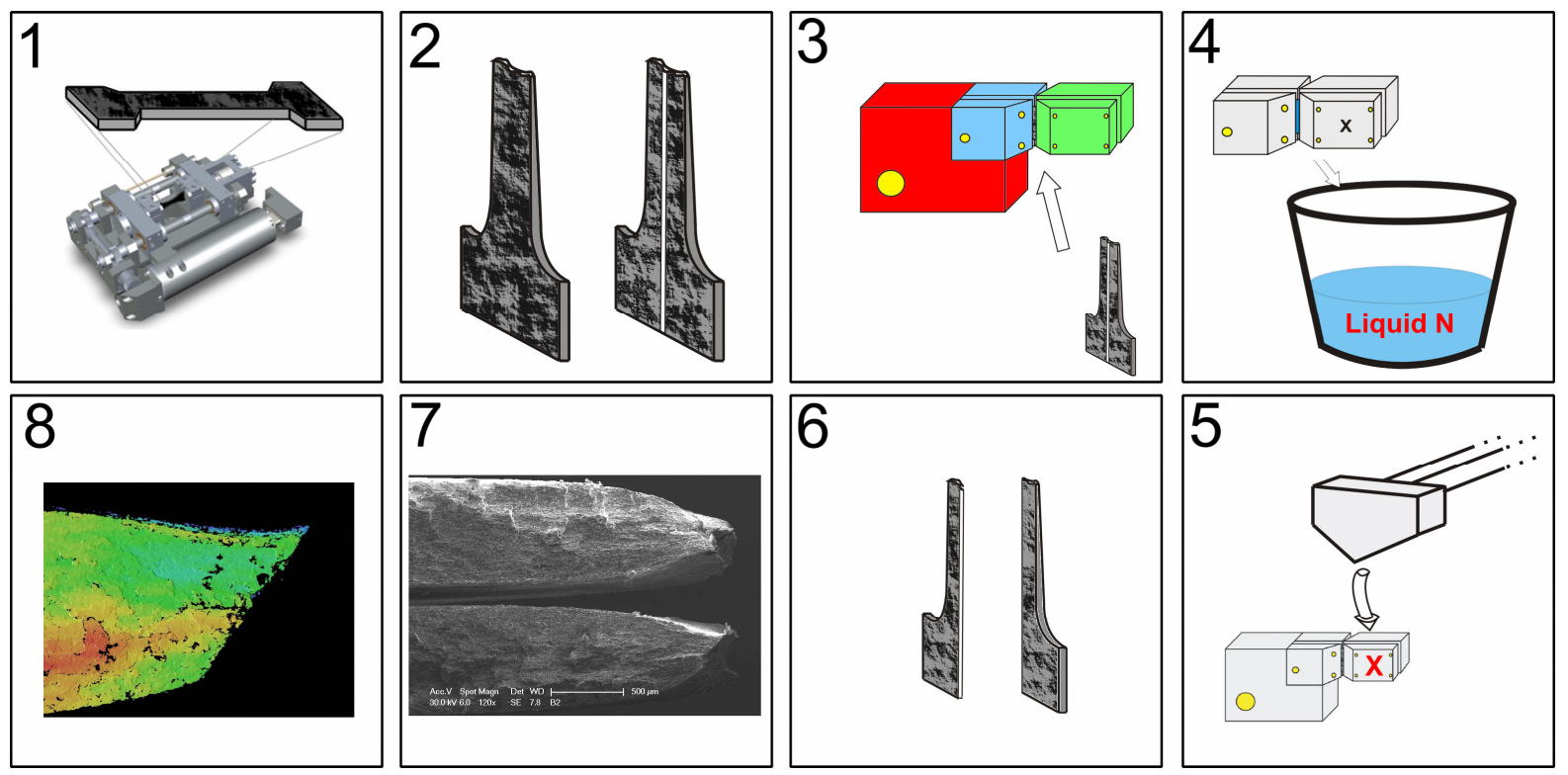

Figure 1. Steps of the experimental methodology to achieve controlled brittle-fracture along a desired cross section. 1) Tensile test with a tensile stage, 2) Placing a groove on the cross section to be investigated, 3) Placing the sample into the specially-designed clamping mechanism, 4) Cooling the clamped specimen in liquid nitrogen for a sufficient amount of time, 5-6) Fracturing the sample using a charpy impact hammer, 7) SEM analysis, 8) Profilometry analysis. 
With this methodology sheet metal specimens tested in any strain path can be opened up, however the verification here is carried out with uniaxial tensile tests (step 1, Fig. 1). The tests are carried out with a tensile stage from Kammrath and Weiss, at a strain rate of $20 \mu \mathrm{m} / \mathrm{sec}$. All tensile tests are carried out in rolling direction.

To increase the stress triaxiality and intensification, a groove is placed on the tested samples, using a rasor blade (step 2, Fig. 1). The width of the groove is approximately 100mu and the depth is 30mu (Fig. 2). Afterwards, the specimen is placed in a specially designed clamping mechanism (step 3, Fig. 1). This mechanism consists of three separate parts, which are represented with different colors in Fig. 1. The red part is a bulk, stationary component which can be fixed on a charpy-impact tester. The blue part, which grips the specimen from one side, can be fitted to the red part with two thick pins (not shown in the figure), which allows for a fast connection. The green part, grips the other side of the specimen, and is not connected to the blue part.

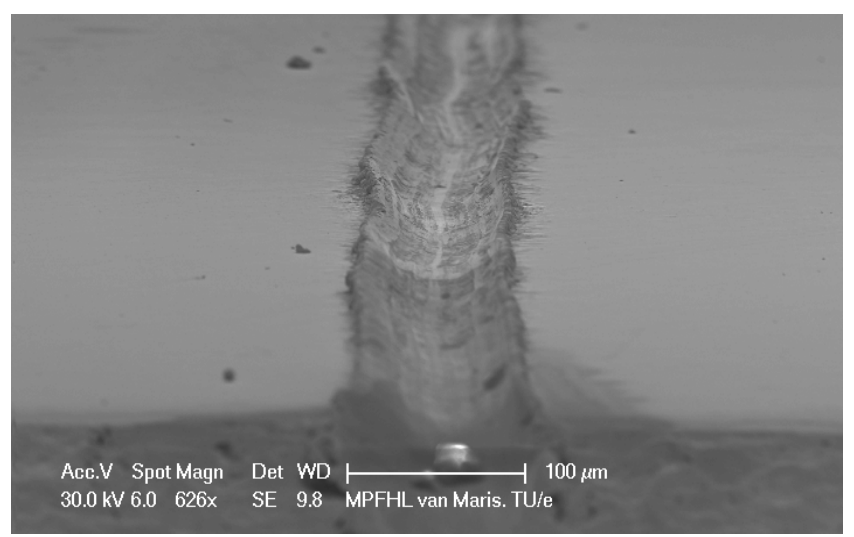

Figure 2. The groove that is placed on the sample to be brittle-fractured

Before moving on to the test itself, the specimen has to be cooled down below its ductile-to-brittle transition temperature. This is achieved by placing the blue and green parts of the clamping mechanism, which are connected to each other by the specimen itself, in a container of liquid nitrogen (step 4, Fig. 1). After a sufficient period of time ( $\sim$ half an hour), the clamp is taken out and swiftly placed back on the red part (through the pins). The charpy hammer is then released to hit the green part of the clamping mechanism (step 5, Fig. 1). Brittle fracture is more favorable under tensile stresses, while shear stresses are more likely to induce plastic deformation and therefore ductile fracture. Accordingly, to achieve an ideal stress state for brittle fracture, the charpy hammer is aimed at the edge of the green part, at a location far away from the sample. This imposes a large moment, and the resulting tensile stresses (which is further intensified by the notch itself) enhances the chances of having a brittle fracture.

To determine the applicability of this methodology for analysing deformation-induced effects on the metal microstructures, three steels of different mechanical characters (e.g. formability, strength), interstitial-free steel(IF), re-phosporized interstitial-free steel (IFr) and dual-phase steel (DP) are tested.

To investigate the revealed microstructures, metallographic examination is carried out comparatively on both surfaces of the fractured samples (step 7, Fig. 1) with a scanning electron microscope (Philips XL 30 ESEM-FEG). Furthermore, profilometric analysis is also carried out using a dual head confocal-laser interferometer (Sensofar) to determine the exact dimensions of microstructural features in the studied materials (step 8, Fig. 1). 


\section{Results}

Materials. The tensile test results of the tested steels are given in Fig 3. It is clearly seen that IF steel is more formable than both IFr and DP steels, probably due to its low-carbon content and clean (interstitial-free) microstructure. IFr steel, which is a variation of the same class of steels, has a relatively higher tensile strength than IF steel (due to re-phosphorization) in expense of some formability. Dual-phase steel on the other hand has a much higher tensile strength than both IF and IFr (probably due to the martensite content) but a relatively limited elongation to fracture.

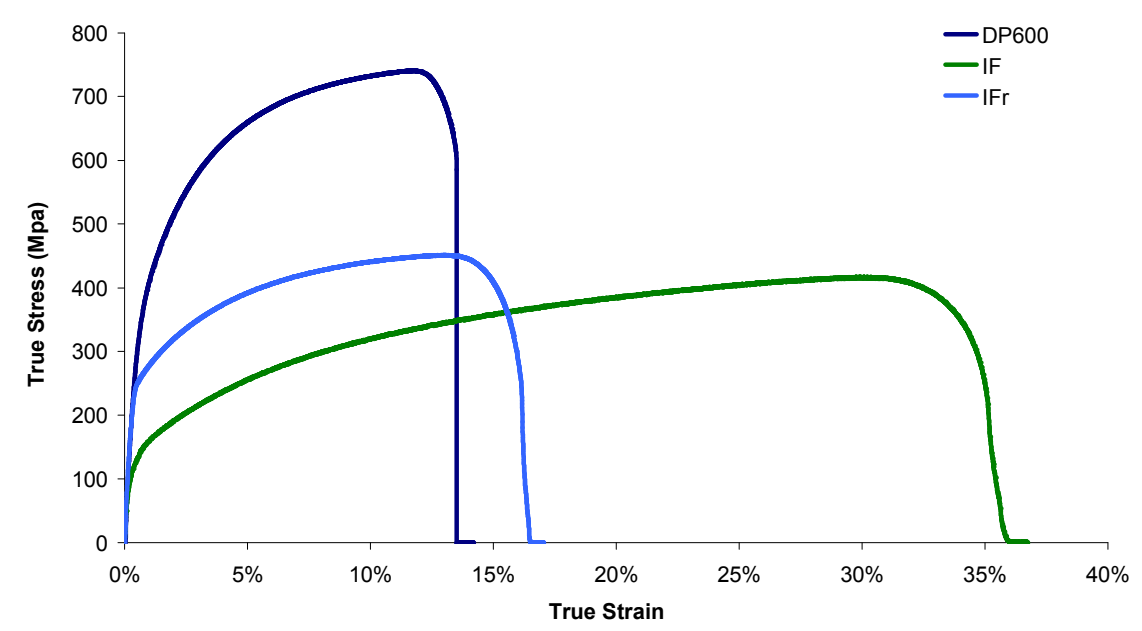

Figure 3. Tensile performance of the sheet metals used in this work.

Methodology. We start with our analysis by commenting on the achievability of brittle fracture using the developed setup. Fig. 4 shows two images of the cross sections of as-received (i.e. not tensile tested) dual phase steel, revealed by the brittle fracture method. The optical micrograph shows the "shiny" surface obtained (Fig. 4(a)), while the SEM image shows an example of the classical cleavage steps observed on this sample, both of which are indications of brittle fracture. Similar results are also obtained with IF and IFr steels, however, it should be mentioned that especially in the case of the IF steel minor modifications to the methodology (i.e. longer cooling period, deeper notch) are found to be necessary. Both of these modifications indicate that in the case of the highly formable IF steel more severe conditions (i.e. lower temperature, more triaxial compared to shear stress state, and higher strain rate) are necessary to make brittle fracture favorable over shear fracture.
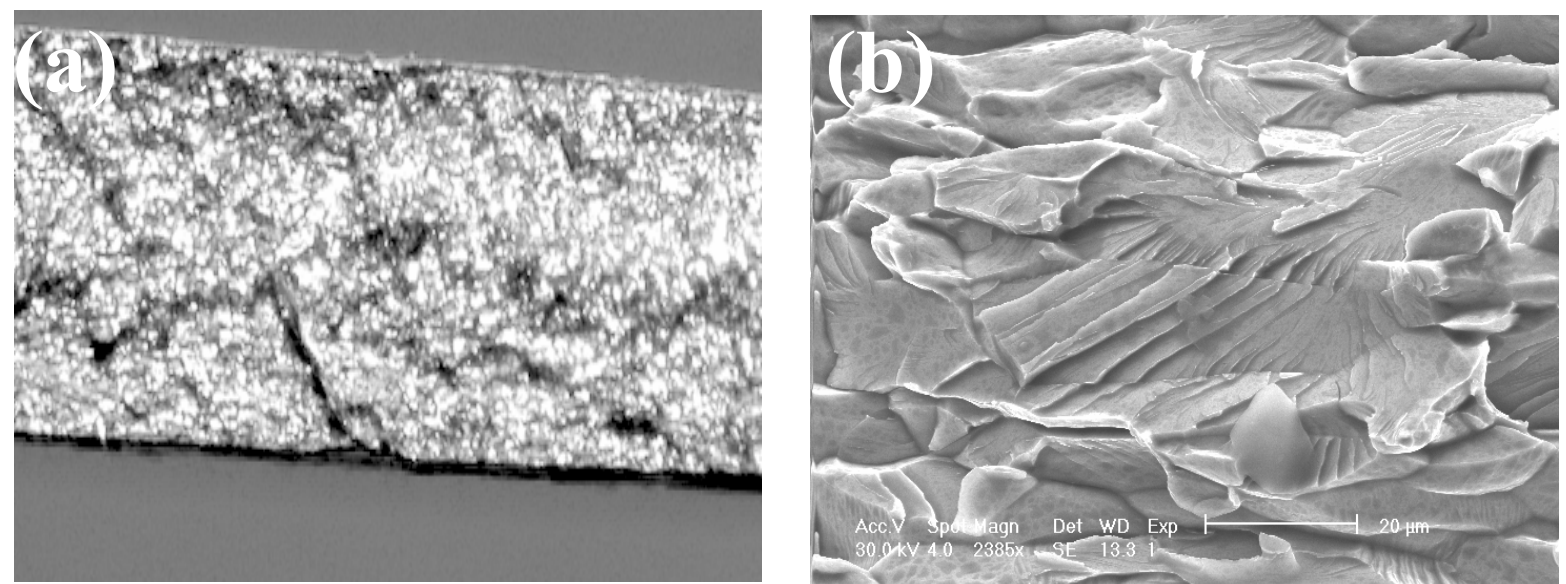

Figure 4. Images of the as-received microstructure of DP steel with (a) light microscopy, and (b) electron microscopy. 
Moving on to the tested samples, where a strong strain gradient exists due to the tensile test, an interesting observation is made regarding the observed mode of the brittle failure. In different regions of these specimens it is possible to see both transgranular and intergranular fracture morphologies. For example, for the IF steel, the brittle fracture is generally transgranular (Fig. 5(a)) in the undeformed part of the tensile specimen (i.e. clamp region) and away from the neck. However, near the neck of the tensile test specimens, intergranular crack propagation is observed to be more favourable among the severely deformed and elongated grains (Fig. 5(b)). Although not shown here due to space limitations, similar behaviours are observed in IFr and DP600 steels, although in these specimens a few cleavage sites are also encountered together with the intergranular fracture sites near the neck region.

As intergranular brittle fracture is not observed in the cross sections of the as-received specimens (Fig. 4(b)) the mode of the brittle fracture is probably affected by the strain gradient existing on the tensile test samples. Digital image correlation analysis shows e.g. for IF steel that, local strains up to $160 \%$ are reached during a tensile test near the fracture zone. At such high levels of local deformations, grains are elongated extensively and the grain boundaries are severely disrupted. Upon the exertation of a high level of tensile force at low temperatures, such weak spots are more probable to be the initiation sites for brittle cracks. The observation that some cleavage sites do exist in the neck regions of the IFr and DP steels also strengthen this analysis, as these steels fail at much lower levels of total elongations (Fig. 3) compared to the IF steel, leading to relatively lower deformation of grain boundaries.
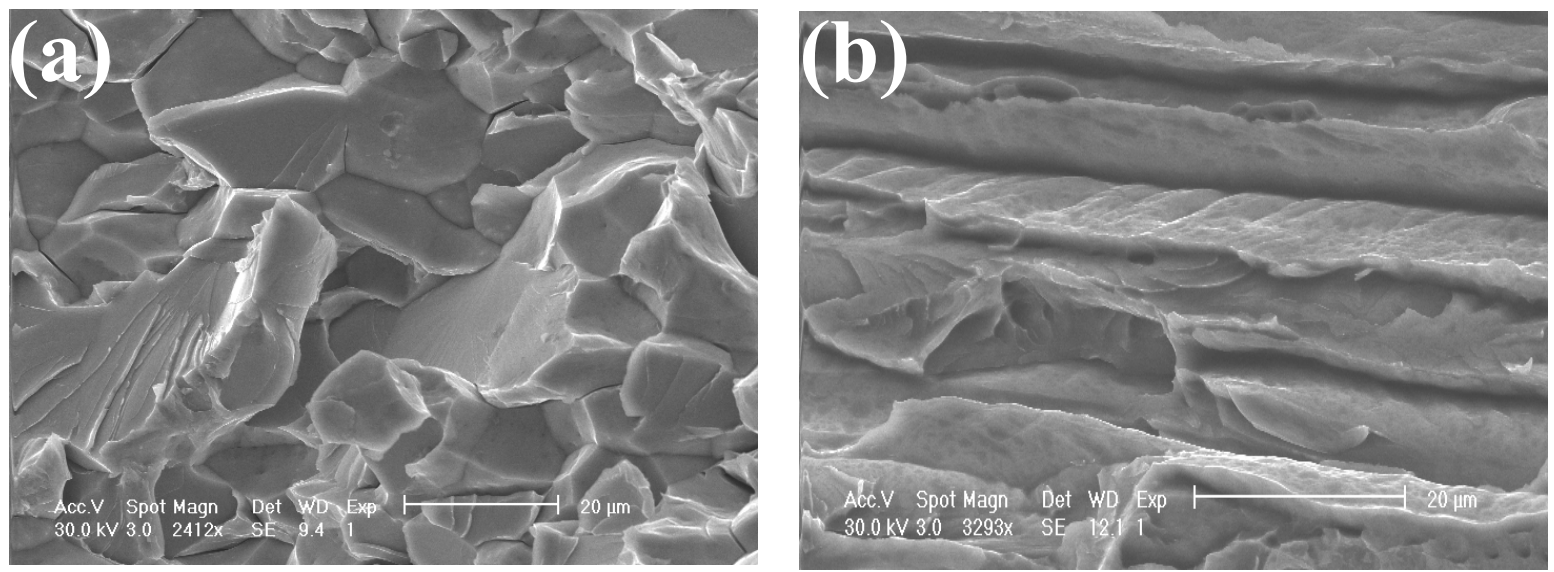

Figure 5. Different brittle fracture modes in different regions of the tested IF specimen, (a) Cleavage away from the neck (b) Intergrannular fracture near the neck.

Characterization. The SEM micrographs of the tensile test samples prepared by the brittle fracture methodology are given in Fig 5 and 6. As suggested in the introduction part, a much more representative picture of the underlying microstructure is achieved with this methodology, compared to the conventional methods. In Fig. 6(a), for example, the neck region of the IF specimen is shown. The 3D nature of the grains that are elongated towards the fracture surface can be clearly observed. Furthermore, high magnification examination not only reveals important details about the geometry and size of these grains but also clarifies the initiation mechanisms and locations of microvoids due to ductile deformation. For IF steel, for example, triple grain boundaries are observed to be probable nucleation sites (Fig. 6(b)). Comparative analysis using both of the obtained surfaces make it possible to visualize the 3D character of such microstructural features, (e.g. grains, voids etc). An example of such a comparison is given in Fig. 7. Note that here, to make visualization easier, the micrograph on Fig. 7(b) is flipped vertically. 

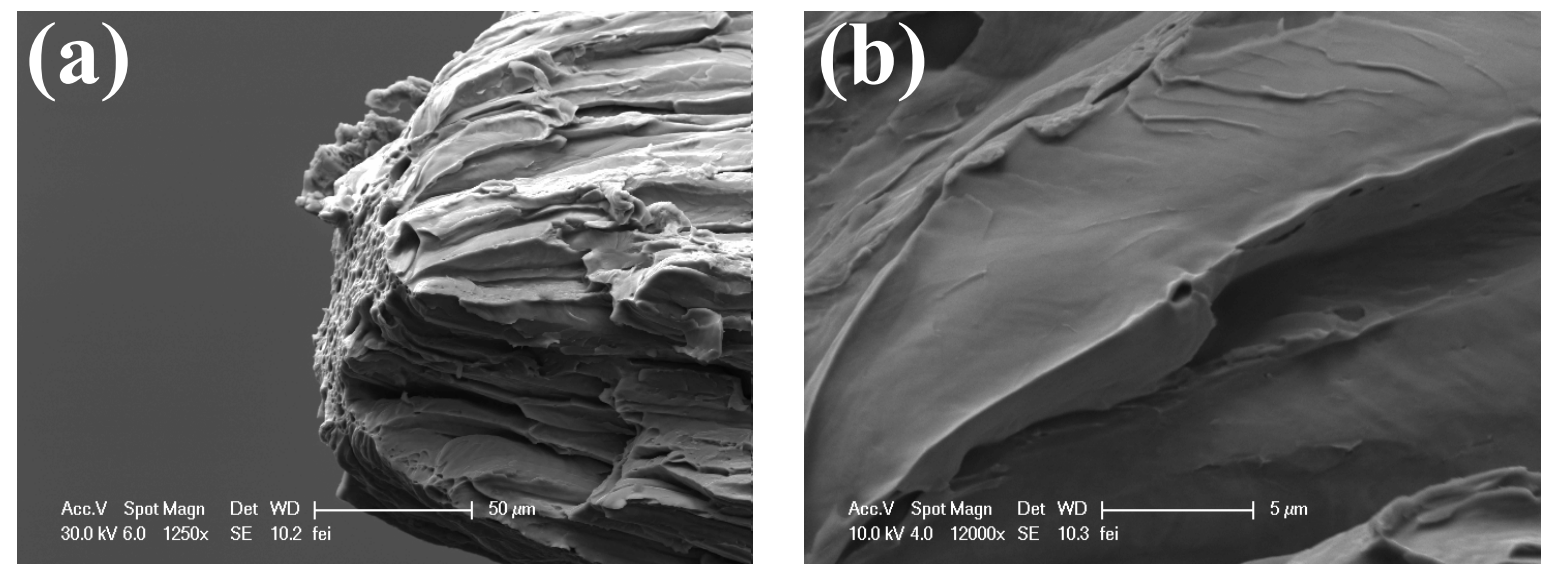

Figure 6. (a) The severely deformed microstructure of the IF steel, near the neck of the tensile test specimen, (b) Microvoid nucleated on the triple point of a grain boundary.
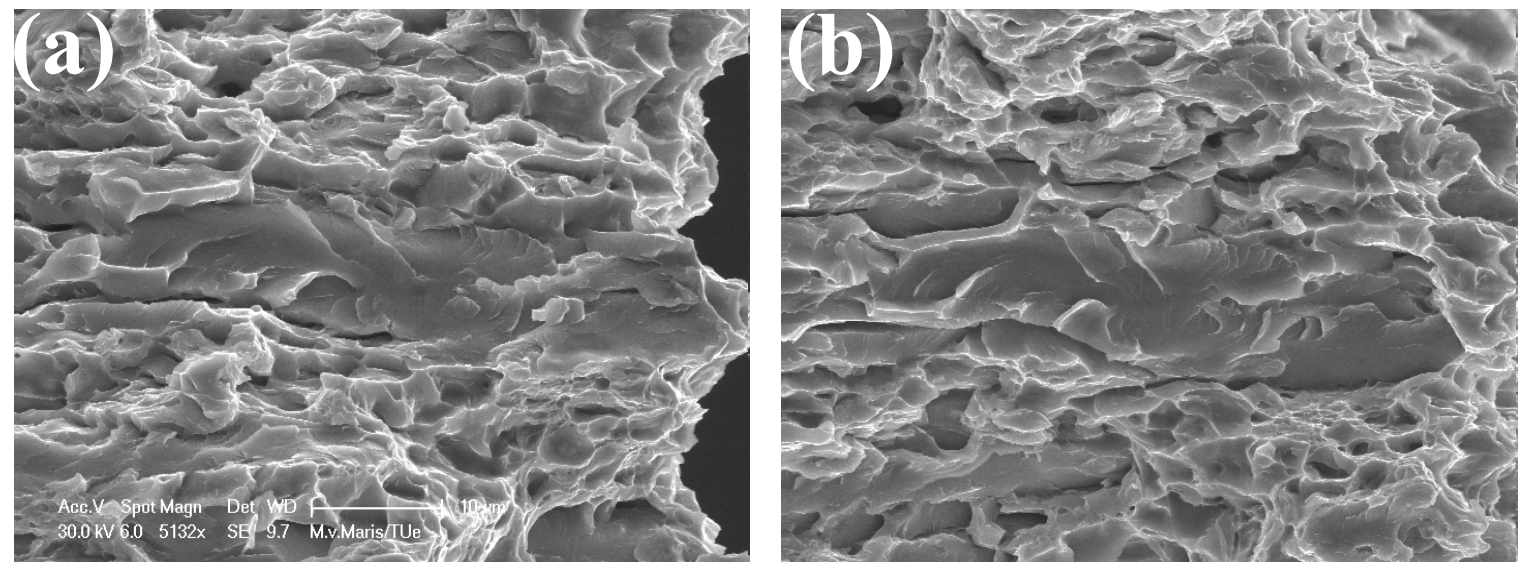

Figure 7. The two sides of the brittle-fractured neck region of DP600 steel. Note that (b) is flipped vertically.

Furthermore, a more quantitative analysis of the microstructure is also possible, for example by the use of confocal microscopy as shown in Fig. 8(b). In this figure, the topographical analysis of the brittle-fractured DP steel specimen (Fig. 8 (a)) is given. Although some regions were not completely retrieved with this analysis (due to the limitations of confocal microscopy at steep slopes), useful information about grain or microvoids dimensions can be obtained, which can later on be used to compare and couple with the data from the other brittle surface, leading to a $3 \mathrm{D}$ representation of the material microstructure at that cross section of interest.
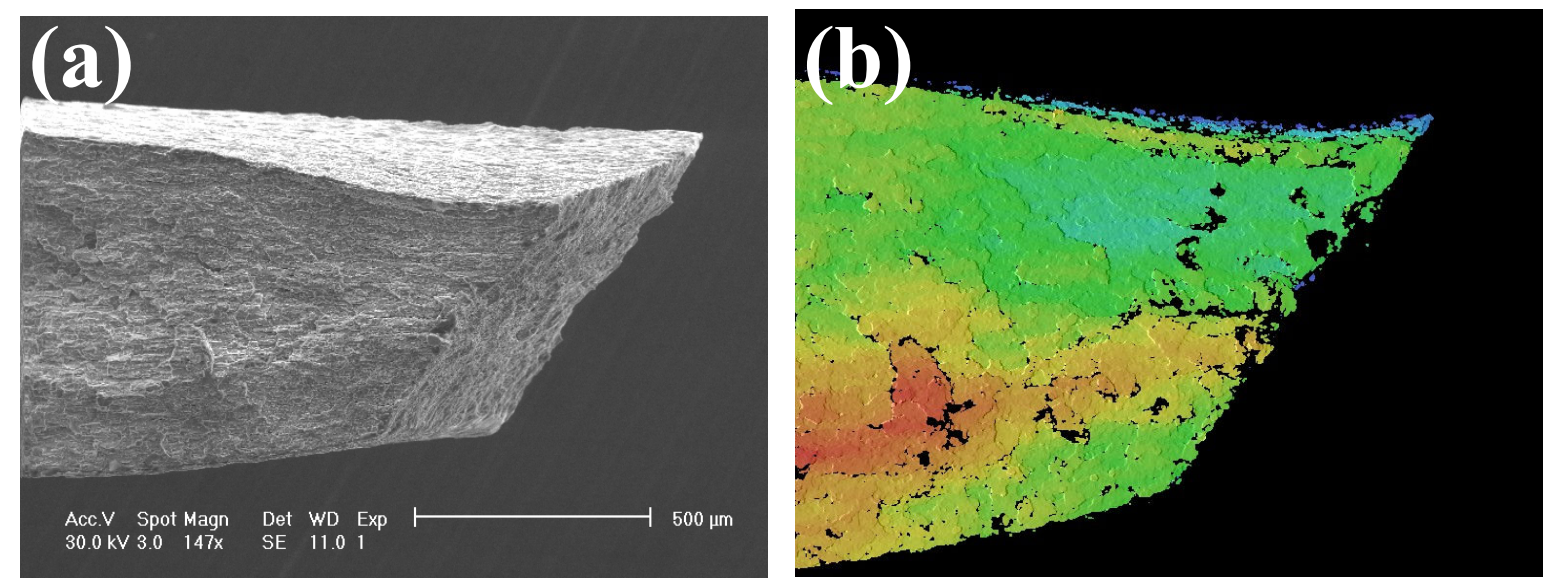

Figure 8. (a) Neck region of the DP steel opened with brittle-fracture method, (b) Topographical analysis of the surface shown in (a). 


\section{Conclusions}

In this work we present an experimental methodology to investigate metal microstructures. This methodology involves opening up fractured metal specimens under the ductile-to-brittle transition temperature to yield two parts in a brittle manner. To achieve this, an experimental setup is developed that not only makes brittle fracture more favorable compared to ductile deformation but also makes it more controllable, such that it can be triggered at any cross section of interest. The occurrence of brittle fracture is validated by analyzing the unaltered microstructure from SEM characterization and surface profilometry is used for further characterization. It is found that this technique yields additional valuable information regarding the size and morphology of deformed grains or nucleation mechanisms of ductile damage. Experiments with a number of different steels show that the methodology can be applied to sheet of different formability.

\section{References}

[1] L.E. Samuels: J. Inst. Met. 85:51-62 (1956/1957)

[2] Y.B. Guo and A.W. Warren, J. Manuf. Sci. Eng. 127 (2005), p. 333

[3] Zhong Z, Hung NP (2002) Grinding of alumina/aluminum composites. J. Mater. Process. Technol. 123(1):13-17

[4] J. Yu, J. Liu, J. Zhang and J. Wu, Mater. Lett. 60 (2006), pp. 206-209

[5] Y.W. Shi and J.T. Barnby, Int. J. Frac. 25, 143 (1984).

[6] Tai, W. H.: Eng. Fract. Mech., 37(4), pp. 853-880 (1990)

[7] G.E. Dieter: Mechanics of Materials (McGraw-Hill, Tokyo 1961). 